\title{
COMBINING AHP WITH DEA FOR MCDM: AN APPLICATION TO SELECTION OF DECISION ALTERNATIVES FOR A HYDROPOWER DEVELOPMENT PROJECT
}

\author{
Ming Li and Hongcai Sun \\ Department of Strategy, Norwegian School of Management \\ Elias Smiths vei 15, Box 580, N-1301 Sandvika, Norway \\ Beijing Institute of Decision Science and Management \\ N-24 Tai Ping Rd., Beijing 100850, P.R. China \\ ming.li@bi.no/ hcs@cenpok.net.cn
}

\begin{abstract}
The paper presents a way is to integrate the DM's value judgements represented by the AHP comparison matrices into the cone-ratio DEA model $\cdot$ by defining its feasible regions on weights of benefits and costs as convex polyhedral cones. The extended model based on such combination has been applied to choose the most desirable alternative for a hydropower development project in China. The application shows that the proposed approach is more reasonable and useful for benefitcost analysis especially when the number of alternatives is large.
\end{abstract}

\section{Introduction}

The Benefit-Cost Analysis (BCA) is widely applied to Multiple Criteria Decision Making (MCDM) problems where benefits are gains and advantages of all sorts - economic, physical, psychological and social while costs are losses and pains of all the same sorts. To make such a decision, the Analytic Hierarchy Process (AHP) is often regarded as a useful analysis tool (Saaty, 1980). One of its outstanding advantages is the well-defined framework for value-focused thinking, since it structures a complex decision problem through a value system, i.e., a criterion or objective hierarchy, that identify relations between criteria and other factors whether intangible or concrete. With respect to a specific BCA situation, a decision-maker (DM) conventionally structures two hierarchies. One is for the benefits where the DM inquires for which alternative yields the greater benefits. The other is for the costs where the DM looks for which alternative incurs the greater costs. Through the ratio of benefits to costs, a priority on performances of a set of decision alternatives can be elicited (Bennett and Saaty, 1993). In this sense, the AHP is usually characterized as a subjective assessment. In practice, however, the DM may find difficult to concentrate on making pairwise comparisons when the alternatives are too many. Even if the DM gave his value judgements, the validity of the relevant comparison matrix would be suspicious. Moreover, if the number of the alternatives is large, the relative priorities of the alternatives would be small and be significantly affected by errors arising out of inconsistency in pairwise comparisons (Saaty, 1994).

In contrast with the AHP, the Data Envelopment Analysis (DEA) can handle a large number of alternatives for BCA. The DEA was put forward to assess the relative efficiencies of homogeneous Decision Making Units (DMUs) which use multiple inputs to 'produce' multiple outputs (Charnes et al., 1978). In DEA, the decision criteria are first ordered in terms of which ones are costs and which ones are benefits. The costs are also called inputs and the benefits outputs. Compared with other BCA methods, DEA uses a more compelling assessment index - the efficiency index - that similar to the ratio of benefits to costs to assess the alternatives. With DEA models, each alternative is allowed to use any set criterion weights that put itself in the best possible light subject to some restrictions. The result is a separation of a set of alternatives in a subset of efficient ones, and a subset of inferior ones. This is useful for MCDM in discriminating between a large number of viable alternatives without using the DM's 
subjective judgements. With such a feature, the DEA may be characterized as an objective assessment. There is now an extensive literature has pointed out the advantages of using the DEA efficiency for assessing alternatives in MCDM problems (Tone, 1988; Belton, 1992, Doyle and Green, 1993; Stewart, 1996). The weight flexibility, however, is also regarded as a problem with the DEA, since it often finds too many efficient solutions, and these solutions often are associated with unacceptable weights. To overcome such problem, alternative approaches of treating factor weights in the DEA models have been developed (Roll and Golany (1993). For solving MCDM problems, however, the restrictions should be determined by the DM with respect to his preferences on relative importance of the decision criteria.

It is natural therefore to combine the AHP with the DEA to make full use of their merits for BCA. In this paper, we propose an approach that integrates the DM's value judgements represented by the AHP comparison matrices into the DEA model. The paper is organized as following: The second section presents a way on how to define weight feasible regions in the cone-ratio DEA model as convex polyhedral cones tensioned by column vectors of the AHP comparison matrices. The third section shows how our approach is used to choose the most desirable alternative from a large number of alternatives for a hydropower development project in China. In the fourth section, a brief conclusion is given.

\section{Combination of the AHP and the DEA}

Regarding the alternatives as virtual DMUs, the BCA for a MCDM problem may be formulated within the DEA framework by taking the alternative's costs as inputs and benefits as outputs. For the $q$ th alternative, the DEA efficiency index $E_{q}$ - a ratio of the sum of its weighted benefits to the sum of its weighted costs - is associated with its overall performance. According to the cone-ratio model (Charnes et al., 1989; Wei and $Y u, 1997)$, the efficiency of the $q$ th alternative with $Y_{q}=\left(y_{1 q}, y_{2 q}, \ldots, y_{s q}\right)^{\mathrm{T}}$ and $X_{q}=\left(x_{1 q}, x_{2 q}, \ldots\right.$, $\left.x_{m q}\right)^{\mathrm{T}}$ is found by solving the.following fractional programming model:

M1:

$$
\operatorname{Max}\left(E_{q}\right)=\frac{u^{\mathrm{T}} Y_{q}}{v^{\mathrm{T}} X_{q}}
$$

Subject to

$$
\begin{aligned}
& \frac{u^{\mathrm{T}} Y_{j}}{v^{\mathrm{T}} X_{j}} \leq 1, \quad j=1,2, \cdots, q, \cdots, n \\
& u \in U, v \in V \text { and } u, v \neq 0
\end{aligned}
$$

where $V$ and $U$ are cones that represent feasible regions of the criterion weights. $V \in E_{+}^{m}, U \in E_{+}^{s}$, $E_{+}^{s}$ and $E_{+}^{m}$ are positive Euclidean vector spaces with $s$ and $m$ dimensions, respectively. $u=\left(u_{1}, u_{2}, \cdots, u_{s}\right)^{\mathrm{T}}$ and $v=\left(v_{1}, v_{2}, \cdots, v_{m}\right)^{\mathrm{T}}$ are weight vectors for the benefit and the cost criteria.

Let $t=\left(v^{\top} X_{q}\right)-1, \omega=t v$ and $\mu=t u$, the model (M1) can be converted into the following equivalent linear programming version:

M2:

$$
\begin{aligned}
& \operatorname{Max}\left(E_{q}\right)=\mu^{\mathrm{T}} Y_{q} \\
& \omega^{\mathrm{T}} X-\mu^{\mathrm{T}} Y \geq 0, \\
& \omega^{\mathrm{T}} X_{q}=1, \\
& \mu \in U, \omega \in V \text { and } \mu, \omega \neq 0 .
\end{aligned}
$$

Subject to

where $Y=\left(Y_{1}, Y_{2}, \cdots, Y_{n}\right)$ is a $s \times n$ matrix for the benefit scores, and $X=\left(X_{1}, X_{2}, \cdots, X_{n}\right)$ is a $m \times n$ matrix for the cost scores. $\mu=\left(\mu_{1}, \mu_{2}, \cdots, \mu_{s}\right)^{\mathrm{T}}$ and $\omega=\left(\omega_{1}, \omega_{2}, \cdots, \omega_{m}\right)^{\mathrm{T}}$ are weight vectors related to the benefit criteria and the cost criteria, respectively.

With the model (M2), the relative efficiency of a specific alternative is measured with respect to the best practice frontier defined by the given alternatives. If the alternative is on the frontier, it is defined as 
efficient. Otherwise, it is inefficient. It is clear that the maximum value of $E_{q}^{*}$ is within the interval $(0,1]$. The $q$ th alternative is inefficient when $E_{q}^{*}<1$. But when $E_{q}^{*}=1$, the $q$ th alternative is efficient. In order to assess all alternatives, the model (M2) is solved for each alternative in turn. Consequently, the set of decision alternatives is divided into a subset of efficient ones and a subset of inferior ones.

Since the cone-ratio model allows for a variety of restrictions to be imposed on the criterion weights through suitable definitions of the cones, the weights can be restricted within the DM's weight preference regions by defining the cones as convex polyhedral cones in the Euclidean vector spaces. In practice, the AHP comparison matrices are ideal forms for representing the DM' value judgments and can.be easily used for structuring the weight preference regions (or the cones).

With respect to $m$ cost-oriented criteria, for instance, the DM may present his preferences on the weights of the criteria in the form of a conventional comparison matrix $C\left(c_{i j}\right): c_{i j}=\omega_{j} / \omega_{j}>0, c_{i j}=1 / c_{i j}(\forall i \neq j)$ and $c_{i i}=1$. The $k$ th column vector $c^{(k)}$ in $C$ can be regarded as a complete pairwise comparison when the $k$ th criterion is chosen as a reference criterion. It is known that in the model (M2) the cone $V$, the preference region on the cost-oriented criteria, can therefore be specifically structured by all of the normalized column vectors $c^{(k)}(k=1,2, \ldots, m)$ in $C$. Moreover, any weight vectors $\omega$ in $V$ can be obtained from $c^{(k)}$ 's linear combinations

$$
\omega=\sum_{k=1}^{m} \lambda_{k} c^{(k)}=C \lambda \in V, \quad\left(\lambda_{k} \geq 0, \forall_{k}\right)
$$

where $\lambda=\left(\lambda_{1}, \lambda_{2}, \ldots, \lambda_{m}\right)^{\mathrm{T}}$ is co-ordinate vector for $\omega$ corresponds to vectors $c^{(1)}, c^{(2)}, \ldots, c^{(m)}$.

The above weight preference region (or the cone $V$ ) has the following features: (1) If $C$ is a unit square matrix, i.e., $c_{i j}=1, c_{i j}=0(\forall i, j=1,2, \cdots, m$ and $i \neq j)$, then either all criteria are incomparable, or for whatever reasons the DM is not able to make pairwise comparisons among the criteria. It implies the DM has no preferences about the criteria, and his preference region could be regarded as a positive Euclidean space constructed by $\mathrm{m}$ unit orthogonal vectors. (2) If $c_{i j}=1(\forall i, j=1,2, \cdots, m)$, then the DM thinks all criteria to be equally important. In this case, the preference region becomes a line through the origin and is the symmetric axis of the positive Euclidean space. (3) If $C$ is a consistent matrix, i.e., the consistency index C.I. $(C)=0$ which means the column vectors in $C$ are linearly dependent on each other, and the preference region becomes a line through the origin in the positive Euclidean space. If C.I. $(C)>0$, then the DM's judgements are inconsistent, which indicates that the preference region is a polyhedral closed convex cone. Therefore, the greater the value of C.I., the more inconsistent the DM's judgement, and the greater the preference region. (4) If the rank $R(C)=r(r=1,2, \ldots, m)$, then there are $r$ linearly independent column vectors in $C$. These vectors are called extreme preference vectors because they stand for all of the DM's extreme preferences on the criterion weights. It is known that the preference region is completely determined by the extreme preference vectors and it is a cone with $r$ edges.

In the same way, the cone $U$ in the model (M2) associated with the preference region on the benefitoriented criteria can be specifically structured by the normalized column vectors $b^{(r)}$ s in a relevant comparison matrix $B$, and any weight vectors $\mu$ in the cone $U$ can be obtained by

$$
\mu=\sum_{r=1}^{s} \varphi_{r} b^{(r)}=B \varphi \in U, \quad\left(\varphi_{r} \geq 0, \forall r\right)
$$

where $\varphi=\left(\varphi_{1}, \varphi_{2}, \ldots, \varphi_{s}\right)^{T}$ is co-ordinate vector for $\mu$ corresponds to vectors $b^{(1)}, b^{(2)}, \ldots, b^{(\mathrm{s})}$.

By substituting $\mu$ and $\omega$ in the model (M2) with the above equalities, the cone-ratio model is converted into the following model formulation:

M3:

$$
\operatorname{Max}\left(E_{q}\right)=\lambda^{\mathrm{T}}\left(B^{\mathrm{T}} Y_{q}\right)
$$

Subject to

$$
\lambda^{\mathrm{T}}\left(C^{\mathrm{T}} X\right)-\varphi^{\mathrm{T}}\left(B^{\mathrm{T}} Y\right) \geq 0
$$




$$
\begin{aligned}
& \lambda^{\mathrm{T}}\left(C^{\mathrm{T}} X_{q}\right)=1 \\
& \varphi \in E_{+}^{s}, \lambda \in E_{+}^{m} \text { and } \varphi, \lambda \neq 0,
\end{aligned}
$$

where $B^{\mathrm{T}} Y=\left(B^{\mathrm{T}} Y_{1}, B^{\mathrm{T}} Y_{2}, \ldots, B^{\mathrm{T}} Y_{n}\right)$, is a $s \times n$ matrix; $C^{\mathrm{T}} X=\left(C^{\mathrm{T}} X_{1}, C^{\mathrm{T}} X_{2}, \ldots, C^{\mathrm{T}} X_{n}\right)$, is a $m \times n$ matrix. The model (M3) is called as the V-D model because it combines the value judgements with the DEA model.

There are basic conclusions for this model (their proofs are omitted due to the length of the paper):

Theorem 1. If alternative $q$ is efficient under the V-D model (M3), then it is also efficient under the model (M2). But not vice versa.

Theorem 2. For any reciprocal comparison matrix $C$, the principal right eigenvector $w_{\lambda}$ belongs to the preference region constructed by the normalized column vectors in $C$.

Corollary. When using the DEA efficiency index to rank the alternatives, the first ranked alternative from AHP must be one of the efficient alternatives from the V-D model.

\section{An Application of the V-D Model}

The approach is applied to a MCDM problem where the decision goal is to choose the most desirable alternative project from 27 alternatives that were generated for developing a hydropower station in China. There are six decision criteria: $b_{1}$ - electricity production $\left(\mathrm{GWh} /\right.$ year); $b_{2}$ - flood control(million $\mathrm{m}^{3} /$ year) $; b_{3}$ - transportation improvement (a nominal scale from 0 to 10 , and 10 is the best); $c_{1}$ production costs (million $\$ /$ year) ; $c_{2}$ - environmental impacts (a nominal scale from 0 to 10 , and 10 is the worst); $c_{3}$ - evacuated villages (in number). The scores of some alternative projects are listed in Table1. The DM is a group including the president of SinoPower (an energy company in China), the mayor of Yibin city in Sichuan province and an administrator from Sichuan Environment Protection Bureau (SEPB).

Table 1. Criteria and relevant attributes for decision

\begin{tabular}{ccccccc}
\hline Alternative & \multicolumn{3}{c}{ The Cost-oriented Criteria } & \multicolumn{3}{c}{ The Benefit-oriented Criteria } \\
Projects & $c_{1}$ & $c_{2}$ & $c_{3}$ & $b_{1}$ & $b_{2}$ & $b_{3}$ \\
\hline P1 & 8.2 & 7 & 5 & 9.0 & 7.1 & 5 \\
P2 & 4.6 & 2 & 2 & 6.8 & 9.4 & 6 \\
$\ldots$ & $\ldots$ & $\ldots$ & $\ldots$ & $\ldots$ & $\ldots$ & $\ldots$ \\
P14 & 5.6 & 3 & 3 & 5.8 & 9.4 & 6 \\
P15 & 6.3 & 7 & 1 & 9.3 & 6.8 & 8 \\
$\ldots$ & $\ldots$ & $\ldots$ & $\ldots$ & $\ldots$ & $\ldots$ & $\ldots$ \\
P26 & 4.8 & 4 & 2 & 5.2 & 6.7 & 8 \\
P27 & 8.5 & 6 & 6 & 8.6 & 7.5 & 4 \\
\hline
\end{tabular}

A two-step procedure is proposed in the application. In the first step, an initial assessment will be conducted by using the model (M2) to divide all alternatives into efficient ones and inefficient ones. The assessment in the first step may be called as an objective estimation since the DM's value judgements are not employed yet. In the second step, further discrimination will be made in order to find out which alternative is the most desirable one. The assessment in the second step may be regarded as a subjective discrimination since the V-D model is used.

Table 2 shows the model (M2)'s solutions in which P2, P15 and P26 are efficient while others are not. It can be seen that the decision problem can not be solved so far. Firstly, there are still three alternative projects can offer the best practice, and the DM had to determine which one is the most desirable one. Secondly, the weights assigned to the criteria were unacceptable from the viewpoint of the DM, since some weights are actually zero and the relative importance between criteria implied by the weights were not in agreement with the DM's value judgements. 
Table 2. Initial solutions by using the model (M2)

\begin{tabular}{cccccccc}
\hline & $\begin{array}{c}\text { Efficiency } \\
\text { Index }\end{array}$ & \multicolumn{2}{c}{ Weights for the Cost-oriented Criteria } & \multicolumn{3}{c}{ Weights for the Benefit-oriented Criteria } \\
& $\omega_{1}$ & $\omega_{2}$ & $\omega_{3}$ & $\mu_{1}$ & $\mu_{2}$ & $\mu_{3}$ \\
\hline P1 & 0.7425 & 0.0699 & 0.0001 & 0.0001 & 0.0473 & 0.0001 & 0.0001 \\
P2 & 1.0000 & 0.0362 & 0.0833 & 0.0833 & 0.0245 & 0.0177 & 0.0278 \\
$\ldots$ & $\ldots$ & $\ldots$ & $\ldots$ & $\ldots$ & $\ldots$ & $\ldots$ & $\ldots$ \\
P14 & 0.8214 & 0.0980 & 0.0001 & 0.0001 & 0.0001 & 0.0480 & 0.0001 \\
P15 & 1.0000 & 0.0265 & 0.0238 & 0.1667 & 0.0180 & 0.0245 & 0.0208 \\
$\ldots$ & $\ldots$ & $\ldots$ & $\ldots$ & $\ldots$ & $\ldots$ & $\ldots$ & $\ldots$ \\
P26 & 1.0000 & 0.0565 & 0.0166 & 0.0812 & 0.0128 & 0.0099 & 0.0459 \\
P27 & 0.6844 & 0.0698 & 0.0001 & 0.0001 & 0.0473 & 0.0001 & 0.0001 \\
\hline
\end{tabular}

For further discrimination between the efficient alternative projects, the DM made value judgements in the form of the comparison matrices $B$ and $C$ associated with the benefit-oriented criteria and the costoriented criteria, respectively

$$
C=\left[\begin{array}{ccc}
1 & \frac{1}{3} & 2 \\
3 & 1 & \frac{1}{2} \\
\frac{1}{2} & 2 & 1
\end{array}\right], B=\left[\begin{array}{ccc}
\frac{1}{1} & 4 & 2 \\
\frac{1}{4} & 1 & 3 \\
\frac{1}{2} & \frac{1}{3} & 1
\end{array}\right]
$$

By introducing the comparison matrices into the V-D model (M3), the alternative projects are then assessed with respect to the DM's weight preference regions on the criteria. Table 3 shows the valued efficiencies of the alternatives and the weights related to the extreme preference vectors. From the results, only P2 keeps efficient while $\mathrm{P} 15$ and $\mathrm{P} 26$ become inefficient. So that $\mathrm{P} 2$ is the most desirable alternative project for developing the hydroelectric power station.

Table 3 . The final results of the assessment and the criteria weights Efficiency Weights for the Cost-oriented Criteria

Weights for the Benefit-oriented Criteria

\begin{tabular}{cccccccc} 
& Index & $\omega_{1}$ & $\omega_{2}$ & $\omega_{3}$ & $\mu_{1}$ & $\mu_{2}$ & $\mu_{3}$ \\
\hline P1 & 0.7425 & 0.3151 & 0.9451 & 0.1576 & 0.2845 & 0.4261 & 0.1421 \\
P2 & 1.0000 & 0.4106 & 1.0419 & 0.2657 & 0.3207 & 0.2639 & 0.1058 \\
$\ldots$ & $\ldots$ & $\ldots$ & $\ldots$ & $\ldots$ & $\ldots$ & $\ldots$ & $\ldots$ \\
P14 & 0.7006 & 0.4727 & 1.4179 & 0.2364 & 0.1249 & 0.0315 & 0.0623 \\
P15 & 0.9846 & 0.3001 & 0.7616 & 0.1943 & 0.2343 & 0.1928 & 0.0773 \\
$\ldots$ & $\ldots$ & $\ldots$ & $\ldots$ & $\ldots$ & $\ldots$ & $\ldots$ & $\ldots$ \\
P26 & 0.7243 & 0.4209 & 0.6316 & 0.2105 & 0.4885 & 0.7321 & 0.2441 \\
P27 & 0.6756 & 0.2331 & 0.6997 & 0.2330 & 0.2839 & 0.4252 & 0.1418 \\
\hline
\end{tabular}

\section{Conclusion}

The AHP offers an ideal value-focused thinking framework to organize a DM's value systems or goal hierarchies on a variety of decision problems and is easily used for eliciting the DM's preferences on decision criteria. The DEA is a linear programming based technique for measuring the relative efficiency of the decision making units with multiple inputs and outputs and its results can be affected by the DM's judgements on weights (or relative importance) of the inputs and outputs (or cost-oriented criteria and benefit-oriented criteria). The merits of the AHP in the well-defined comparison matrices and the DEA in the weight flexibility can be combined by defining the feasible regions for the weights in DEA model in the form of the AHP comparison matrices. The application of the approach shows that the combination is practical and the so-called V-D model is more reasonable and useful not only for eliciting the DM's preferences on decision criteria, but also for assessing a large number of alternatives within the DM's 
weight preference regions. It should be a promising way for dealing with MCDM problems with considering benefits and costs.

Of course, a further discrimination between efficient alternatives might have to be conducted. This may be progressed by narrowing down the preference regions. There are many ways could be applied to do this. The effectiveness of the potential approaches will be investigated in the following-up research.

\section{References}

Belton, V., (1992), "VIDEA: Integrated DEA and MCDA - A Visual Interactive Approach". Proceedings of the Tenth International Conference on MCDM 4/2, pp.419-429.

Bennett, J.P. and Saaty, T.L., (1993), "Knapsack Allocation of Multiple Resource in Benefit-Cost Analysis by Way of the Analytic Hierarchy Process". Mathl. Comput. Modelling, Vol.17 (4/5)

Charnes, A., Cooper, W.W., and Rhodes, E., (1978), "Measuring the Efficiency of Decision Making Units", European Journal of Operational Research 2/6, 429-444.

Charnes, A., Cooper, W.W., Wei, Q.L. and Huang, Z.M., (1989), "Cone-ratio Data Envelopment Analysis and Multiobjective Programming". Int. J. Syst. Sci., Vol.20, pp.1099-1118.

Doyle, J. and Green, R, (1993), "Data Envelopment Analysis and Multiple Criteria Decision Making". OMEGA Int. J. of Mgmt Sci., Vol.21(6), pp.713-715.

Roll, Y. and Golany, B., (1993), "Alternate Methods of Treating Factor Weights in DEA". OMEGA Int. J. of Mgmt Sci. Vol.21(1), pp.99-109.

Saaty, T.L., (1980), The Analytic Hierarchy Process. New York: McGrow-Hill

Saaty, T.L., (1990), "How to make a decision: The Analytic Hierarchy Process". European Journal of Operational Research, Vol.48, pp.9-26.

Saaty, T.L., (1994), Fundamentals of Decision Making and Priority Theory with the Analytic Hierarchy Process. Pittsburgh: RWS Publications

Stewart, T.J., (1996), "Relationships between Data Envelopment Analysis and Multicriteria Decision Analysis". Journal of the Operational Research Society, Vol.47, pp.654-665

Tone, K., (1988), "A Comparative Study on AHP and DEA". Reprints of the International Symposium on The Analytic Hierarchy Process, pp. 39-43, Tiajin University, Taijin, China

Wei, Q. L. and Yu, G., (1997), "Analyzing Properties of K-cones in the Generalized Data Envelopment Analysis Model". Journal of Econometrics, Vol.80, pp.63-84 